\title{
Numerical Investigation of Mixed Convection Heat Transfer of Nanofluid in a Lid Driven Square Cavity with Three Triangular Heating Blocks
}

\author{
Zoubair Boulahia \\ Hassan II University, \\ Laboratory of Mechanics, \\ Casablanca, Morocco
}

\author{
Abderrahim Wakif \\ Hassan II University, \\ Laboratory of Mechanics, \\ Casablanca, Morocco
}

\author{
Rachid Sehaqui \\ Hassan II University, \\ Laboratory of Mechanics, \\ Casablanca, Morocco
}

\begin{abstract}
The present investigation addressed mixed convection heat transfer of nanofluid in a lid driven square cavity with three triangular heating blocks. Finite volume discretization method with SIMPLE algorithm is employed for solving the twodimensional Navier-Stokes and energy balance equations. The method used is validated against previous works. Two cases were considered depending on the position of three triangular heating blocks. Effects of pertinent parameters such as; position of triangular heating blocks, the Richardson number $(0.1 \leq R i \leq 100)$, the Prandtl number of the pure water $(P r=6.2)$ and the volume fraction of nanoparticles $(0 \leq \varphi \leq 0.05)$ on the flow and Nusselt number are investigated. The results of this study illustrate that, by reducing Richardson number and increasing the volume fraction of nanoparticles, the average Nusselt number increases. It is also found that there is an optimal position of triangular heating blocks where the heat transfer rate is maximized.
\end{abstract}

\section{Keywords}

Mixed convection, Lid driven, Cavity, Triangular block, Nanofluid.

\section{INTRODUCTION}

For many years, mixed convection and heat transfer inside cavity is an important event in the various industries. Mixed convection heat transfer with lid-driven cavity flow is frequently encountered in numerous engineering applications such as cooling of electronic devices, lubrication technologies, heating and cooling nuclear systems of reactors and heat exchangers. The literature includes many investigation relating the topic of mixed convection flow inside an enclosure. Work of Khanafer and Aithal. [3] was concentrated on the effect of mixed convection and heat transfer characteristics in a lid-driven cavity with a circular body inside. The results showed that the average Nusselt number increases with an increase in the radius of the cylinder for various Richardson numbers and the optimal heat transfer results are obtained when placing the cylinder near the bottom wall. Islam et al. [2] performed a numerical study on a liddriven cavity with a heated square blockage. They found that size, location and Richardson number of the heater eccentricities affect the average Nusselt number of heater. Work of Oztop et al. [4] can be mentioned as example of such studies, in which they numerically studied mixed convection in square cavities with two moving walls. Their results suggest that when the vertical walls move upwards in the same direction, the heat transfer decreases significantly compared to when the vertical walls move in opposite directions. Kalteh et al. [1] considered laminar mixed convection of nanofluid in a lid-driven square cavity with a triangular heat source. Their simulations indicate that increasing the volume fraction, the nanoparticles diameter and Reynolds leads to an increase in average Nusselt number. El Abdallaoui et al. [6] performed a numerical simulation of natural convection between a decentered triangular heating cylinder and centered triangular heating cylinder [7] in a square outer cylinder filled with a pure fluid or a nanofluid using the lattice Boltzmann method. The results indicate that the horizontal displacement from the centered position to decentered position leads to a considerable increase of heat transfer at weak Rayleigh and the vertical displacement has most important effect on heat transfer at high values of Rayleigh. Sheikholeslami et al. [5] studied the problem of magnetohydrodynamic natural convection heat transfer of nanofluid in a horizontal cylindrical enclosure with an inner triangular cylinder. They found that the results indicate that Nusselt number increase with increasing Rayleigh number and augmentation of the Hartmann number causes the Nusselt number to decrease. Boulahia et al. [16] studied natural convection heat transfer of the nanofluids in a square enclosure with an inside cold obstacle. The results show that the heat transfer rate inside the enclosure increases by increasing the height of the cold block, the volume fraction of nanoparticles and Rayleigh number.

Pishkar et al. [9] studied mixed convection of nanofluid in a horizontal channel. They found that at low Richardson numbers the heat transfer is increased by enlarging the volume fraction of nanoparticles. In addition, their results showed that at low values of Reynolds number, the effects of Richardson number rising on the heat transfer rate for both the nanofluid and pure fluid are negligible. Oztop et al. [8] investigated mixed convection in a lid driven cavity with a cylindrical blockage inside. The vertical left sidewall was moving up or down. The computation was carried out for wide ranges of Richardson number, inner cylinder diameter, center and location of the inner cylinder. They concluded that the most effective parameter on flow field and temperature distribution was the direction of the moving lid. Chen et al. [15] investigated the natural and mixed convection of $\mathrm{Al}_{2} \mathrm{O}_{3}$ water nanofluids in a square enclosure. They found that the force convection cause better heat transfer than natural convection. Boulahia et al. [20] studied mixed convection of the nanofluids in two-sided lid-driven square cavity with a pair of triangular heating cylinders. Muthtamilselvan and Doh [17] presented a steady state two-dimensional mixed convection in a lid-driven square cavity filled with $\mathrm{Cu}$-water nanofluid. They found that Richardson number and solid volume fraction affect the fluid flow and heat transfer in the 
cavity. Moumni et al. [14] studied the nanofluid mixed convection in two-sided lid driven cavity including discrete heat Sources by using the finite volume method. The results showed that by increasing Richardson and Reynolds numbers and the solid volume fraction, the heat transfer rate increases. Boulahia and Sehaqui [18] studied a natural convection of nanofluid in a square cavity including a square heater. They found that by increasing size of the heater, the heat transfer rate enhances.

The aim of this study is to explore the heat transfer rate of mixed convection in a lid driven cavity with three heated triangular cylinders. The first case under investigation is characterized the numerical models used in our study. The computational procedure elaborated in this study is validated against the numerical results of other investigations. We studied the effects of position of the three heating triangular blocks on the heat transfer fluid flow. Wide range of parameters such as Richardson number $(0.1 \leq \mathrm{Ri} \leq 100)$, and volume fraction $(0 \leq \varphi \leq 0.05)$ have been used. The new models of the thermal conductivity and effective

viscosity investigated by Corcione et al. [10] are used to estimate themophysical proprieties of the nanofluid. Our numerical results are presented in the form of plots of isotherms, streamlines and average Nusselt numbers to show the influence of nanofluid and pertinent parameters.

\subsection{Problem Statement}

The problem under investigation is a lid driven square cavity with three heated triangular blocks inside. A schematic diagram of the cavity with triangular cylinders is shown in Fig. 1. The cavity is a square with the height of $\mathrm{H}$, while the size of triangular block is $\mathrm{W}$. The Triangular block is maintained at a temperature $\mathrm{T}_{\mathrm{h}}=310 \mathrm{~K}$. The vertical walls are cooled at constant temperatures $\mathrm{T}_{\mathrm{c}}=290 \mathrm{~K}$, while the horizontal walls are adiabatic. It is assumed that the nanofluid is newtonian, incompressible and laminar and the base fluid and the nanoparticles are in a thermal equilibrium state. The thermo-physical properties of the nanofluid used in this study are evaluated at the average fluid temperature $\left(\mathrm{T}_{\mathrm{c}}+\mathrm{T}_{\mathrm{h}}\right) / 2$ as listed in Table 1.

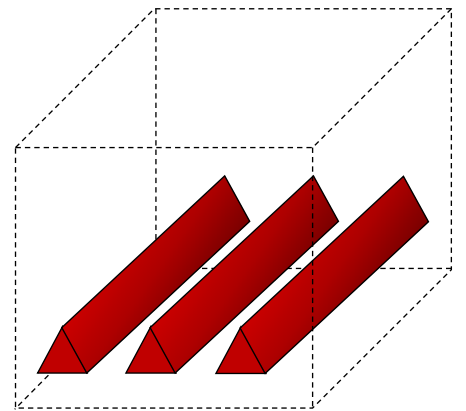

Case 1
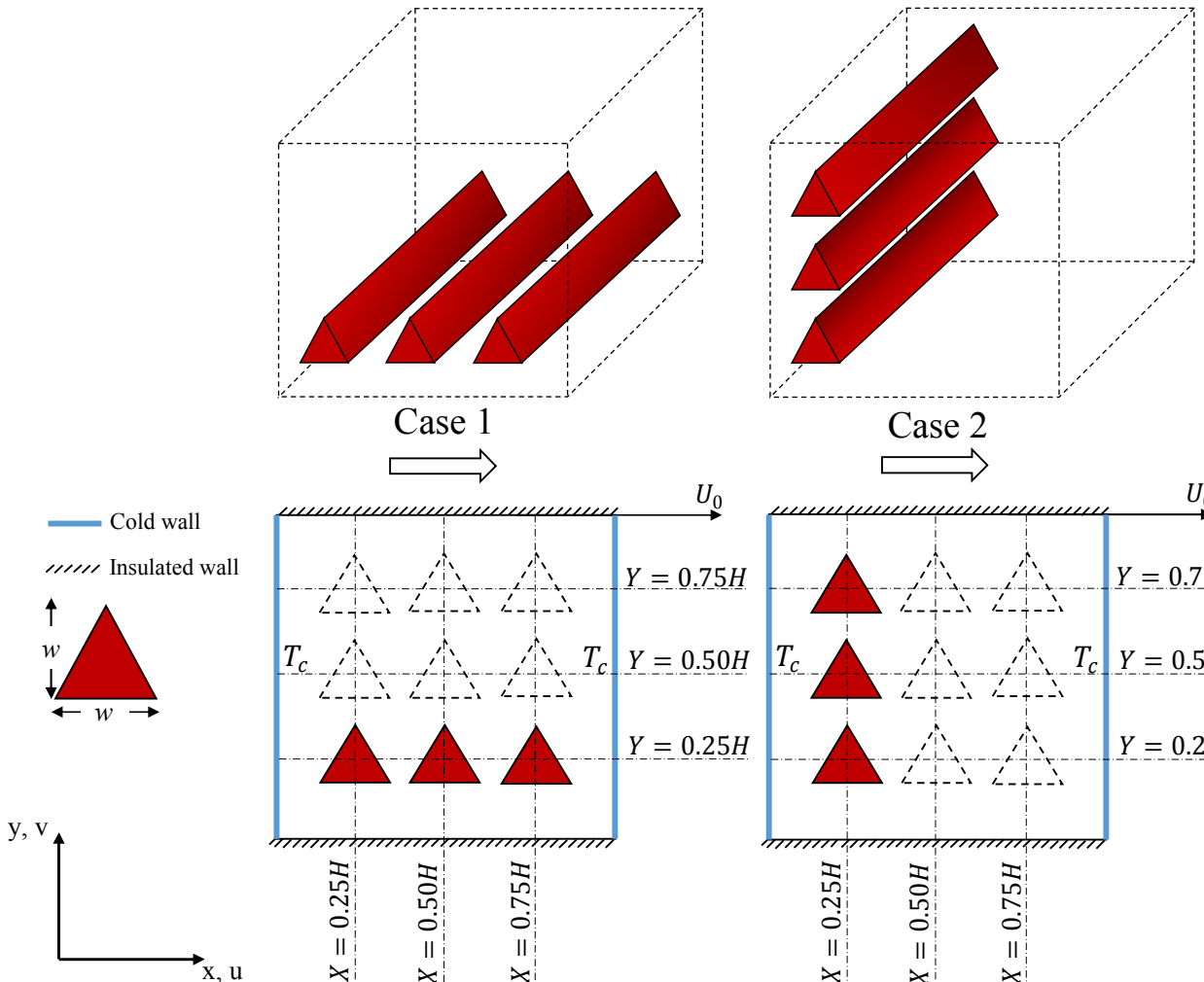

Case 2

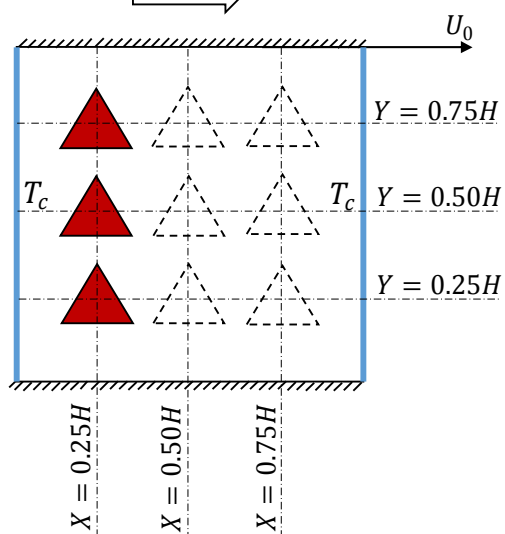

Fig. 1. Schematic of the cavity with three triangular heating sources and boundary conditions.

Table 1. Thermo-physical properties of water and nanoparticles at $\mathbf{T}=\mathbf{3 0 0} \mathrm{K}[21]$

\begin{tabular}{lll}
\hline & Copper $(\mathrm{Cu})$ & Water $\left(\mathrm{H}_{2} \mathrm{O}\right)$ \\
\hline$C_{p}(\mathrm{~J} / \mathrm{Kg} \mathrm{K})$ & 385 & 4179 \\
$\rho\left(\mathrm{Kg} / \mathrm{m}^{3}\right)$ & 8933 & 997.1 \\
$k(\mathrm{~W} / \mathrm{mK})$ & 401 & 0.6 \\
$\beta\left(\mathrm{K}^{-1}\right)$ & $1.6710^{-5}$ & $27.610^{-5}$ \\
$\mu\left(\mathrm{kg} \mathrm{m}^{-1} \mathrm{~s}^{-1}\right)$ & - & $0.85510^{-3}$ \\
\hline
\end{tabular}

\section{MATHEMATICAL FORMULATION}

The governing equations including the two-dimensional transient equations of the continuity, momentum and energy for an incompressible flow are expressed in the following format:

$\frac{\partial u}{\partial x}+\frac{\partial v}{\partial y}=0$

$\frac{\partial u}{\partial t}+u \frac{\partial u}{\partial x}+v \frac{\partial u}{\partial y}=-\frac{1}{\rho_{n f}} \frac{\partial p}{\partial x}+\frac{\mu_{n f}}{\rho_{n f}}\left(\frac{\partial^{2} u}{\partial x^{2}}+\frac{\partial^{2} u}{\partial y^{2}}\right)$ 


$$
\begin{aligned}
\frac{\partial \mathrm{v}}{\partial \mathrm{t}}+\mathrm{u} \frac{\partial \mathrm{v}}{\partial \mathrm{x}}+\mathrm{v} \frac{\partial \mathrm{v}}{\partial \mathrm{y}}= & -\frac{1}{\rho_{\mathrm{nf}}} \frac{\partial \mathrm{p}}{\partial \mathrm{y}}+\frac{\mu_{\mathrm{nf}}}{\rho_{\mathrm{nf}}}\left(\frac{\partial^{2} \mathrm{v}}{\partial \mathrm{x}^{2}}+\frac{\partial^{2} \mathrm{v}}{\partial \mathrm{y}^{2}}\right) \\
& +\frac{(\rho \beta)_{\mathrm{nf}}}{\rho_{\mathrm{nf}}}\left(\mathrm{T}-\mathrm{T}_{\mathrm{c}}\right) \\
\frac{\partial \mathrm{T}}{\partial \mathrm{t}}+\mathrm{u} \frac{\partial \mathrm{T}}{\partial \mathrm{x}}+\mathrm{v} \frac{\partial \mathrm{T}}{\partial \mathrm{y}}= & \alpha_{\mathrm{nf}}\left(\frac{\partial^{2} \mathrm{~T}}{\partial \mathrm{x}^{2}}+\frac{\partial^{2} \mathrm{~T}}{\partial \mathrm{y}^{2}}\right)
\end{aligned}
$$

Where the nanofluid effective density, heat capacity, thermal expansion coefficient and thermal diffusivity are calculated from the following equations [12-11]:

$\rho_{\mathrm{nf}}=(1-\varphi) \rho_{\mathrm{f}}+\varphi \rho_{\mathrm{s}}$

$\left(\rho \mathrm{C}_{\mathrm{p}}\right)_{\mathrm{nf}}=(1-\varphi)\left(\rho \mathrm{C}_{\mathrm{p}}\right)_{\mathrm{f}}+\varphi\left(\rho \mathrm{C}_{\mathrm{p}}\right)_{\mathrm{s}}$

$(\rho \beta)_{\mathrm{nf}}=(1-\varphi)(\rho \beta)_{\mathrm{f}}+\varphi(\rho \beta)_{\mathrm{s}}$

$\alpha_{\mathrm{nf}}=\mathrm{k}_{\mathrm{nf}} /\left(\rho \mathrm{C}_{\mathrm{p}}\right)_{\mathrm{nf}}$

Corcione model [12-10] for dynamic viscosity and thermal conductivity of the nanofluid are given by:

$\mu_{\mathrm{nf}}=\mu_{\mathrm{f}} /\left(1-34.87\left(\mathrm{~d}_{\mathrm{p}} / \mathrm{d}_{\mathrm{f}}\right)^{-0.3} \varphi^{1.03}\right)$

$\frac{\mathrm{k}_{\mathrm{nf}}}{\mathrm{k}_{\mathrm{f}}}=1+4.4 \operatorname{Re}_{\mathrm{B}}^{0.4} \operatorname{Pr}^{0.66}\left(\frac{\mathrm{T}}{\mathrm{T}_{\mathrm{fr}}}\right)^{10}\left(\frac{\mathrm{k}_{\mathrm{p}}}{\mathrm{k}_{\mathrm{f}}}\right)^{0.03} \varphi^{0.66}$

$\operatorname{Re}_{\mathrm{B}}=\frac{\rho_{\mathrm{f}} \mathrm{u}_{\mathrm{B}} \mathrm{d}_{\mathrm{p}}}{\mu_{\mathrm{f}}}$

$\mathrm{u}_{\mathrm{B}}=\frac{2 \mathrm{k}_{\mathrm{b}} \mathrm{T}}{\pi \mu_{\mathrm{f}} \mathrm{d}_{\mathrm{p}}^{2}}$

The boundary conditions for mixed convection written as:

$\mathrm{u}=0, \quad \mathrm{v}=0, \quad \partial \mathrm{T} / \partial \mathrm{y} \quad$ on bottom wall of the $=0 \quad$ cavity

$\mathrm{u}=\mathrm{U}_{0}, \quad \mathrm{v}=0, \quad \partial \mathrm{T} / \partial \mathrm{y} \quad$ on upper wall of the $=0 \quad$ cavity

$\mathrm{u}=0, \quad \mathrm{v}=0, \quad \mathrm{~T}=\mathrm{T}_{\mathrm{c}}$ on right wall of the cavity

$\mathrm{u}=0, \quad \mathrm{v}=0, \quad \mathrm{~T}=\mathrm{T}_{\mathrm{c}}$ on left wall of the cavity

The following dimensionless variables for mixed convection are defined based on properties of pure fluid:

$$
\begin{gathered}
\tau=\frac{t}{\frac{\mathrm{H}}{\bar{U}_{\text {ref }}}}, \quad X=\frac{x}{H}, \quad Y=\frac{y}{H}, \quad U=\frac{u}{U_{\text {ref }}}, \\
V=\frac{v}{U_{\text {ref }}}, \quad P=\frac{p}{\rho_{\text {nf }} U_{\text {ref }}^{2}}, \quad \theta=\frac{T-T_{c}}{T_{h}-T_{c}},
\end{gathered}
$$

Where $\mathrm{U}_{\text {ref }}$ is considered to be $\mathrm{U}_{0}$ for mixed convection. Dimensionless numbers for the system are defined as:

$$
\begin{gathered}
\mathrm{Re}=\frac{\mathrm{u}_{\mathrm{ref}} \mathrm{H}}{v_{\mathrm{nf}}}, \quad \mathrm{Ri}=\frac{\mathrm{Gr}}{\mathrm{Re}^{2}}, \quad \mathrm{Gr} \\
=\frac{\mathrm{g} \beta_{\mathrm{f}}\left(\mathrm{T}_{\mathrm{h}}-\mathrm{T}_{\mathrm{c}}\right) \mathrm{H}^{3}}{v_{\mathrm{f}}^{2}}, \\
\mathrm{Ra}=\text { Gr. Pr }=\frac{\mathrm{g} \beta_{\mathrm{f}}\left(\mathrm{T}_{\mathrm{h}}-\mathrm{T}_{\mathrm{c}}\right) \mathrm{H}^{3}}{\alpha_{\mathrm{f}} v_{\mathrm{f}}}, \quad \operatorname{Pr}=\frac{v_{\mathrm{f}}}{\alpha_{\mathrm{f}}},
\end{gathered}
$$

The governing equations (1)-(4) are written in the following dimensionless form:

$$
\begin{aligned}
& \frac{\partial \mathrm{U}}{\partial \mathrm{X}}+\frac{\partial \mathrm{V}}{\partial \mathrm{Y}}=0 \\
& \frac{\partial U}{\partial \tau}+U \frac{\partial U}{\partial X}+V \frac{\partial U}{\partial Y}=-\frac{\partial P}{\partial X} \\
& +\frac{1}{\operatorname{Re}} \frac{\rho_{\mathrm{f}}}{\rho_{\mathrm{nf}}} \frac{\mu_{\mathrm{nf}}}{\mu_{\mathrm{f}}}\left(\frac{\partial^{2} \mathrm{U}}{\partial \mathrm{X}^{2}}+\frac{\partial^{2} \mathrm{U}}{\partial \mathrm{Y}^{2}}\right) \\
& \frac{\partial \mathrm{V}}{\partial \tau}+\mathrm{U} \frac{\partial \mathrm{V}}{\partial \mathrm{X}}+\mathrm{V} \frac{\partial \mathrm{V}}{\partial \mathrm{Y}}=-\frac{\partial \mathrm{p}}{\partial \mathrm{Y}} \\
& +\frac{1}{\operatorname{Re}} \frac{\rho_{\mathrm{f}}}{\rho_{\mathrm{nf}}} \frac{\mu_{\mathrm{nf}}}{\mu_{\mathrm{f}}}\left(\frac{\partial^{2} \mathrm{~V}}{\partial \mathrm{X}^{2}}+\frac{\partial^{2} \mathrm{~V}}{\partial \mathrm{Y}^{2}}\right) \\
& +\operatorname{Ri} \frac{(\rho \beta)_{\mathrm{nf}}}{\rho_{\mathrm{nf}} \beta_{\mathrm{f}}} \theta \\
& \frac{\partial \theta}{\partial \tau}+U \frac{\partial \theta}{\partial \mathrm{X}}+\mathrm{V} \frac{\partial \theta}{\partial \mathrm{Y}}=\frac{1}{\operatorname{Re} \cdot \operatorname{Pr}} \frac{\alpha_{\mathrm{nf}}}{\alpha_{\mathrm{f}}}\left(\frac{\partial^{2} \theta}{\partial \mathrm{X}^{2}}+\frac{\partial^{2} \theta}{\partial \mathrm{Y}^{2}}\right)
\end{aligned}
$$

Dimensionless form of the boundary conditions can be written as:

$$
\begin{array}{lll}
\mathrm{U}=0, \quad \mathrm{~V}=0, \quad \begin{array}{c}
\partial \theta / \partial \mathrm{y} \\
=0
\end{array} & \begin{array}{l}
\text { on bottom wall of } \\
\text { the cavity }
\end{array} \\
\mathrm{U}=1, \quad \mathrm{~V}=0, \quad \begin{array}{l}
\partial \theta / \partial \mathrm{y} \\
=0
\end{array} & \begin{array}{l}
\text { on upper wall of the } \\
\text { cavity }
\end{array} \\
\mathrm{U}=0, \quad \mathrm{~V}=0, \theta=0 & \begin{array}{l}
\text { on right wall of the } \\
\text { cavity } \\
\text { on left wall of the } \\
\text { cavity }
\end{array}
\end{array}
$$

The total mean Nusselt number of all cavity's wall is defined as:

$\overline{\mathrm{Nu}}_{\text {tot }}=\frac{1}{\mathrm{H}} \int_{0}^{\mathrm{H}} \frac{\mathrm{k}_{\mathrm{nf}}(\varphi)}{\mathrm{k}}\left\{\left|\frac{\partial \theta}{\partial \mathrm{X}}\right|_{\text {left }}+\left|\frac{\partial \theta}{\partial \mathrm{X}}\right|_{\text {right }}\right\} \mathrm{dY}$

\section{NUMERICAL DETAILS}

The calculations are done using the FORTRAN program. A finite volume formulation, given by Patankar [22] on a staggered grid, is applied for discretization of the governing equations (Eqs. (17)-(20)) and boundary conditions described by Eq. (21). SIMPLE (Semi-Implicit Method for Pressure Linked Equations) is used to solve the coupled pressurevelocity equation while Hybrid Differencing Scheme (HDS) of Spalding [19] is used for the convective terms. Line by line application of TDMA (Tri-Diagonal Matrix Algorithm) method [22] is applied on equation systems until sum of the residuals became less than $10^{-6}$.

Table 2. Effect of the grid size on $\overline{N u}_{t o t}$ for the cavity filled with the $\mathrm{Cu}$-water nanofluid $(\varphi=0.05)$ with three triangular heat sources, for $W=w / H=0.2$ and position $X=x / H=0.25$ (see Fig. 1(Case 2))

\begin{tabular}{lllll}
\hline$R i$ & $63 \times 63$ & $83 \times 83$ & $103 \times 103$ & $123 \times 123$ \\
\hline 0.1 & 21.201 & 21.453 & 21.514 & 21.515 \\
100 & 9.844 & 10.071 & 10.132 & 10.135
\end{tabular}

\subsection{Grid independence study}

To find the proper grid size for the numerical simulation, a lid driven square cavity filled with $\mathrm{Cu}$-water nanofluid $(\varphi=$ 0.05 ) having the three triangular heat sources with size 
$\mathrm{w}=0.2 \mathrm{H}$ and position $\mathrm{X}=\mathrm{x} / \mathrm{H}=0.25$ is analyzed in two extreme Richardson numbers $(\mathrm{Ri}=0.1$ and 100$)$. The mean Nusselt number obtained using different grid numbers for particular cases is presented in Table 2 . As can be observed from the table, a uniform $103 \times 103$ grid is sufficiently fine for the numerical calculation.

\subsection{Validations}

The computational procedure was validated against various numerical results available in the literature, three different heat convection problems are chosen. The first case is the numerical results of Iwatsu et al. [13] and Oztop et al. [8] for a top heated moving lid and bottom cooled square cavity filled with air $(\operatorname{Pr}=0.71)$. A $100 \times 100$ mesh was used and the computations were done for three different Richardson numbers. Table 3 demonstrates an excellent comparison of the average Nusselt number between the present results and the numerical results found in the literature [13-8] with a maximum discrepancy of about $1.6 \%$. The second case is a mixed convection flow and heat transfer characteristics in a lid-driven cavity with a circular body inside. The third case is a mixed convection flow and heat transfer in a lid-driven cavity with a square heater inside. Fig. 2 illustrates a comparison of the isotherms and streamlines with the results reported by Islam et al [2] and the results reported by Khanafer and Aithal. [3] at Reynolds number $(R e=100)$.

Table 3. Comparison of $\overline{N u}$ at the hot lid between the present results and those reported in the literature

\begin{tabular}{llll}
\hline \multirow{2}{*}{$R i$} & \multicolumn{3}{l}{ Average Nusselt number at the hot lid } \\
\cline { 2 - 4 } & Iwatsu et al. [13] & Oztop et al. [8] & Present \\
\hline 1.0 & 1.34 & 1.30 & $\mathbf{1 . 3 6}$ \\
0.0625 & 3.62 & 3.63 & $\mathbf{3 . 6 8}$ \\
0.01 & 6.29 & 6.34 & $\mathbf{6 . 2 9}$ \\
\hline
\end{tabular}

Khanafer and Aithal [3]

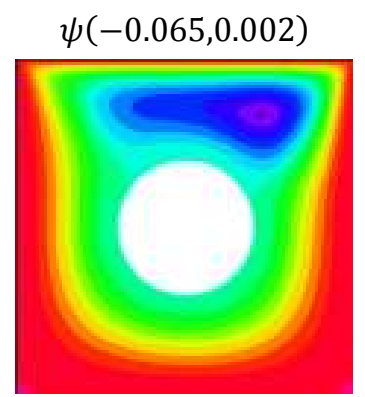

Streamlines $R i=0.01$

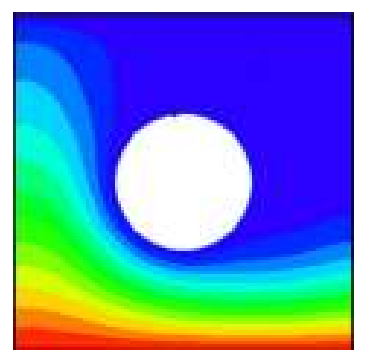

Isotherms $R i=0.01$
Present study

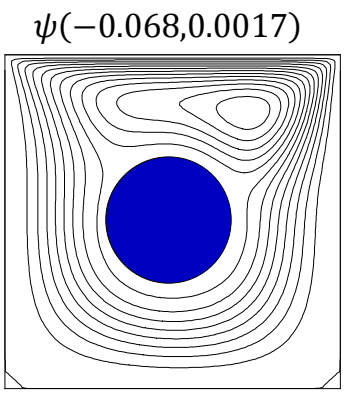

Streamlines $R i=0.01$

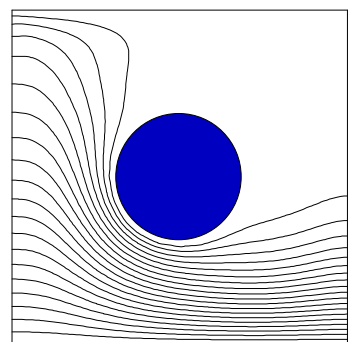

Isotherms $R i=0.01$

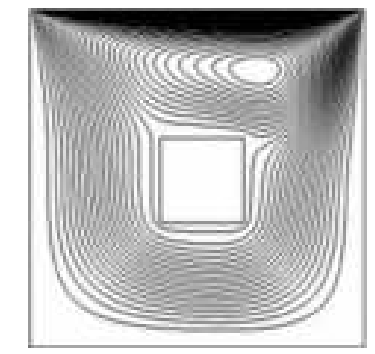

Streamlines $R i=0.1$
Islam et al. [2]

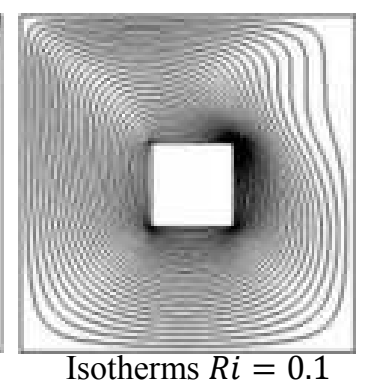

Present study

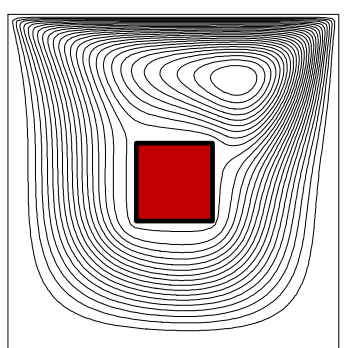

Streamlines $R i=0.1$

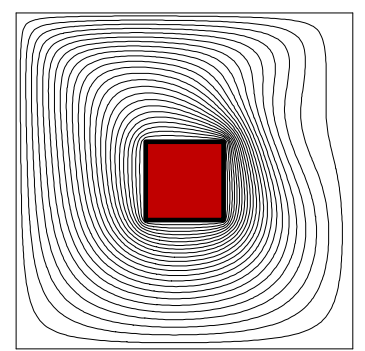

Isotherms $R i=0.1$

Fig. 2. Comparison of present results with the numerical results of Islam et al. [2] $(h / H=0.25, P r=0.7, \operatorname{Re}=100)$ and with results of Khanafer and Aithal [3] $(\operatorname{Re}=100, \mathrm{Pr}=0.7, \mathrm{r} / \mathrm{H}=0.2)$.

\section{RESULTS AND DISCUSSION}

In the present study, numerical results of mixed convection heat transfer of nanofluid in a lid driven square cavity with three triangular heating blocks is numerically simulated. The calculations are carried out in two parts. First, the effect of vertical displacement of three triangular heating blocks $(0.25 \leq \mathrm{Y} \leq 0.75)$ is investigated. In the second part, the calculations are performed for the horizontal displacement of three triangular heating blocks $(0.25 \leq \mathrm{X} \leq 0.75)$ while the Richardson number $(R i)$ is ranging from 0.1 to 100 and the volume fraction of the nanoparticles is varying from 0 to 0.05 . Results of these simulations are presented and discussed in the following two subsections.

\subsection{Vertical displacement of triangular blocks}

In this section, a square cavity filled with $\mathrm{Cu}$-water nanofluid including three triangular heating blocks that were arranged horizontally and positioned vertically from bottom to top $(0.25 \leq \mathrm{Y} \leq 0.75)$ is considered for heat transfer analysis.

Fig.3 (a-b) illustrates the effect of vertical displacement of three triangular heating blocks, the forced convection due to the lid motion and the natural convection due to the buoyancy driven flow, on the streamlines and isotherms for various values of $R i$ ranging from 0.1 to 100 while Grashof number is fixed at $\mathrm{Gr}=10^{4}$. To have a comparative look, the 
streamlines and the isotherms for pure fluid and nanofluid are shown by dashed line and solid line respectively. We can see in Fig. 3, some differences in streamlines and isotherms of pure fluid and nanofluid. We can explain this by the higher viscosity of nanofluid compared to that of the pure fluid which increases the diffusion of momentum in the nanofluid.
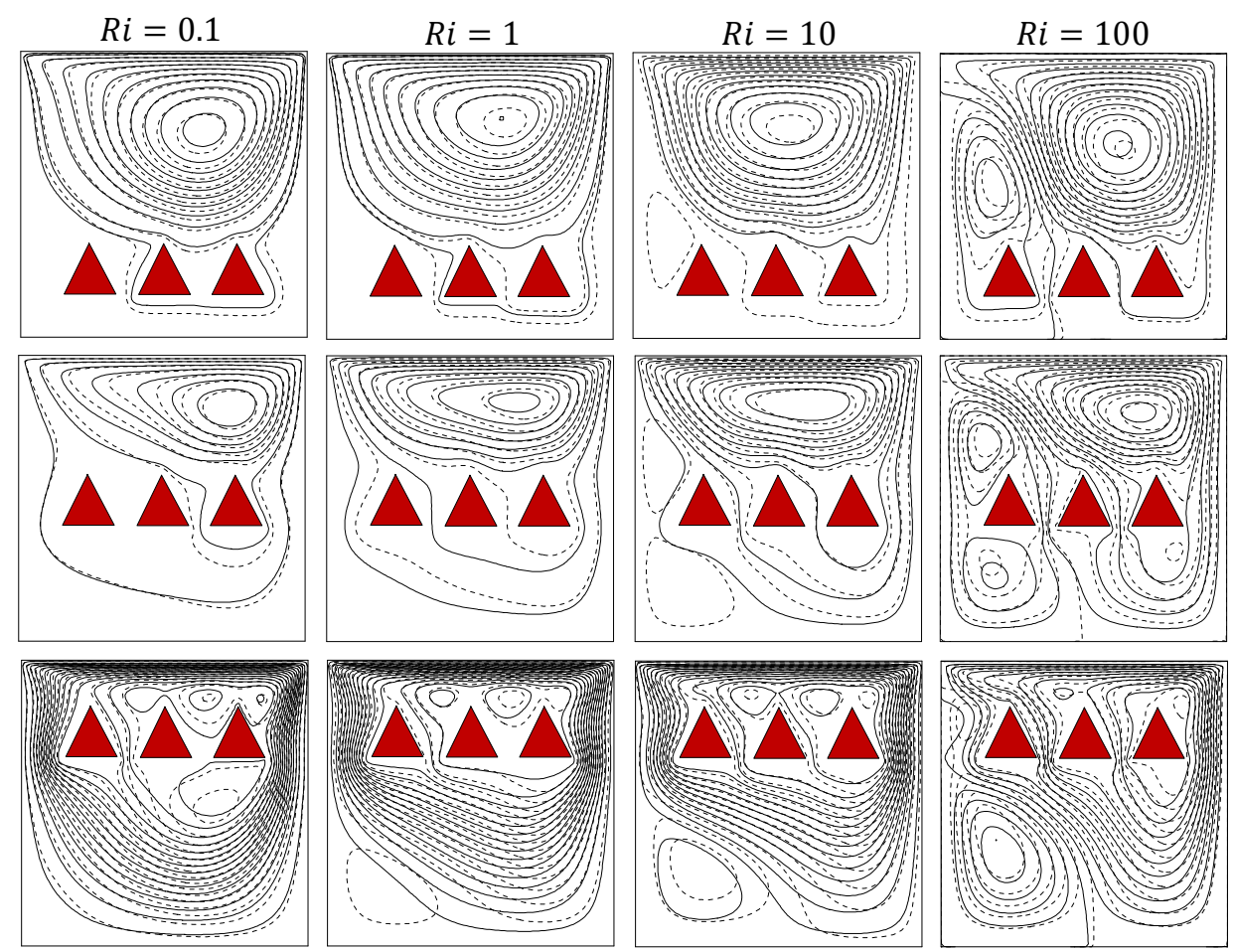

(a) streamlines
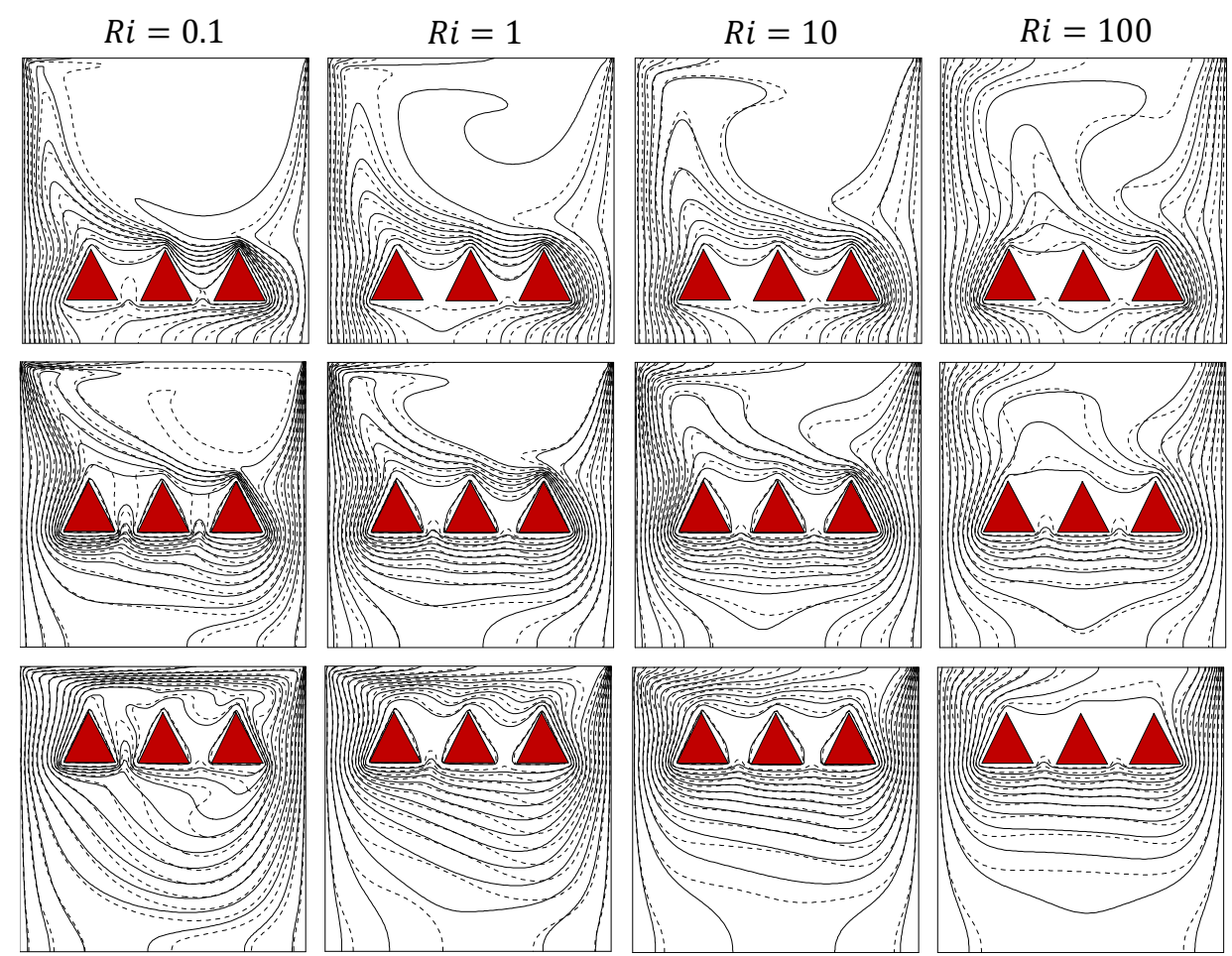

(b) isotherms

Fig. 3. (a) Streamlines and (b) isotherms inside the cavity corresponding to different locations of triangular blocks, i.e. $Y=0.25,0.5$ and 0.75 filled with $\mathrm{Cu}$-water the pure fluid (dashed line) and $\mathrm{Cu}$-water nanofluid (solid line) with $\varphi=$ 5\% and at different Ris. Gr $=10^{4}$.

Fig. 3(a) shows that in the most cases, a clockwise vortex is created inside the cavity under the effect of shear forces. The center of the vortex is changed significantly due to the blocks location, the Ri number variation and buoyancy force effect. At a smaller Richardson number, the flow trend for the rightward lid motion alone is a clockwise rotating vortex with 
its center slightly shifted towards the top right corner. The shear force is more dominant than buoyancy force in the forced convection mode especially when the triangular blocks are located in the lower half of the cavity. This observation shows the higher forced convection effects in the right upper corner of the cavity. As it is clear, the fluid flow under the triangular heated block is very low compare to the upper which is affected by the lid motion. Isotherms in Fig. 3(b) show that at low Ri the concentration of the isotherms, close to the triangular heated blocks increases, which indicates that the heat transfer is through forced convection. By increasing the $R i$, we can see a small counterclockwise rotating eddy started to appear close to the left wall of the cavity (Fig. 3(a)). At $R i=100$, the uniformly distributed isotherms show that the main heat transfer mechanism is occurring through the natural convection. (a)

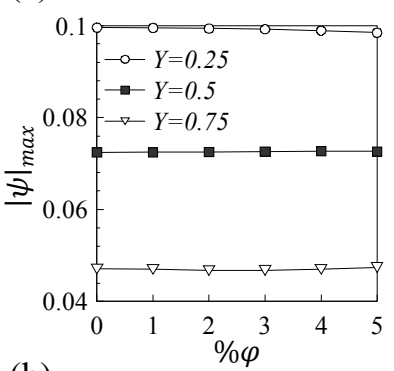

(b)

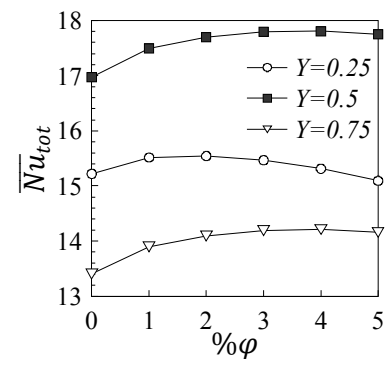

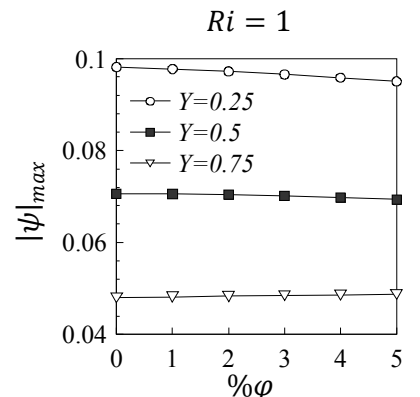

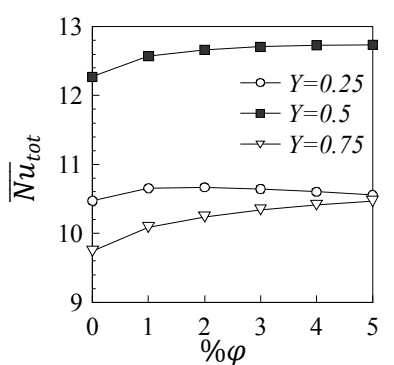

$R i=10$
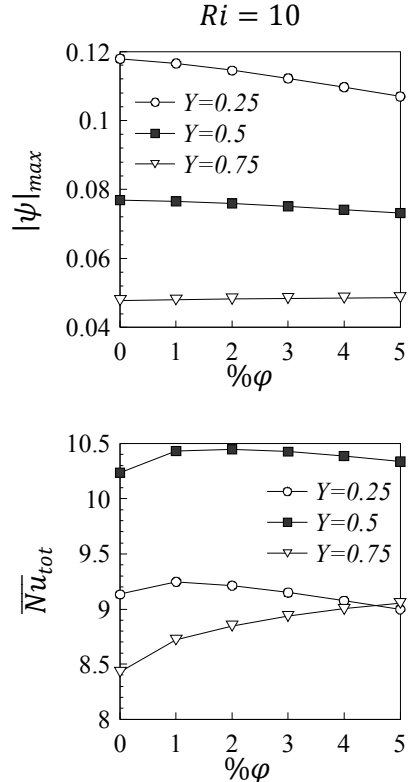
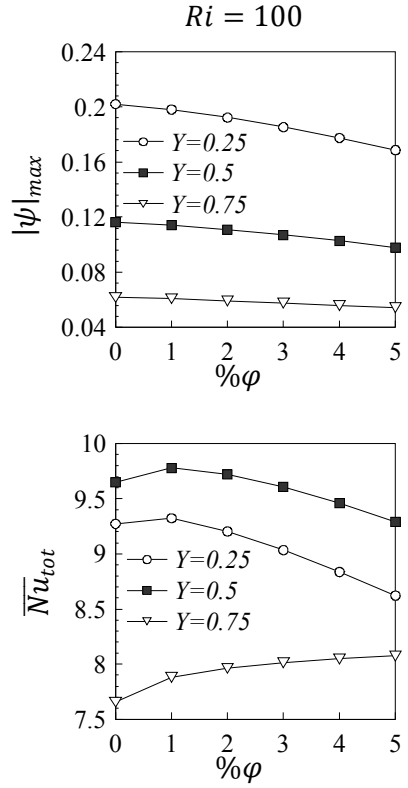

Fig. 4. Variation of (a) $\psi_{\max }$ and (b) $\overline{N u}_{t o t}$ corresponding to different locations of triangular blocks, i.e. $Y=0.25,0.5$ and 0.75 for different Richardson numbers and volume fraction of the nanoparticles. $G r=10^{4}$.

Fig. 4 shows values of $\psi_{\max }$ and $\overline{\mathrm{Nu}}_{\text {tot }}$ in the same conditions of Fig. 3 at different blocks locations, Richardson number and volume fraction of nanoparticles. The maximum stream function $\Psi_{\max }$ shows in Fig. 4(a) decreases at all Ri number by increasing volume fraction of nanoparticles, we can explain the decrease of $\Psi_{\max }$ by Eq. (9) which indicates that the increasing volume fraction of the nanoparticles, leads to increase in the viscosity of the fluid. At low Richardson number (forced convection are dominant), the maximum stream function does not change significantly. Fig. 4(b) shows that changing the blocks location has significant effect on the heat transfer rate. The maximum Nusselt number between these cases $(\mathrm{Y}=0.25,0.5$ and 0.75$)$ occurs in case $(\mathrm{Y}=0.5)$ where there is more space between the triangular blocks and horizontal walls of the cavity which result in better circulation of the flow and hence improvements of convection heat transfer. It is clear that by decreasing the Richardson number and increasing the volume fraction of nanoparticles the rate of convective heat transfer is increased. At high Richardson number $(R i \geq 10)$ the effects of natural convection are dominant so there is an optimum volume fraction of nanoparticles which maximize the heat transfer is about $1 \%$ in the most cases.

\subsection{Horizontal displacement of triangular blocks}

In this section, a square cavity filled with $\mathrm{Cu}$-water nanofluid including three triangular heating blocks that were arranged vertically and positioned horizontally from left to right $(0.25 \leq X \leq 0.75)$ is considered for heat transfer analysis.

When the blocks are located in the left position, we can see from the streamlines of Fig. 5(a) that the flow consists, of a main cell created on the right side of the vertical median of the triangular blocks under the effect of shear forces. At small Richardson number $(\mathrm{Ri} \leq 10)$ (forced convection), the clockwise vortex is mostly generated by the horizontal moving lid while its center slightly shifted towards the top right corner. By increasing Richardson number beyond 10, the effects of moving wall decreases due to the enhancement in the buoyancy force.

When the triangular blocks were arranged vertically on the vertical median $(X=0.5)$, we can see clearly in Fig.5(a), at $R i=100$ that The flow structure is organized into two convection cells, one clockwise vortex on the right and one counterclockwise vortex on the left. This interesting flow is a direct consequence of dominating natural convection at $R i=$ 100. We can see in Fig.5 (b), that by decreasing $R i$ the concentration of the isotherms, close to the triangular heated blocks increases and their patterns are characterized by a complex form. The complex distributed isotherms at small Richardson number show that the main heat transfer mechanism is through the forced convection. 

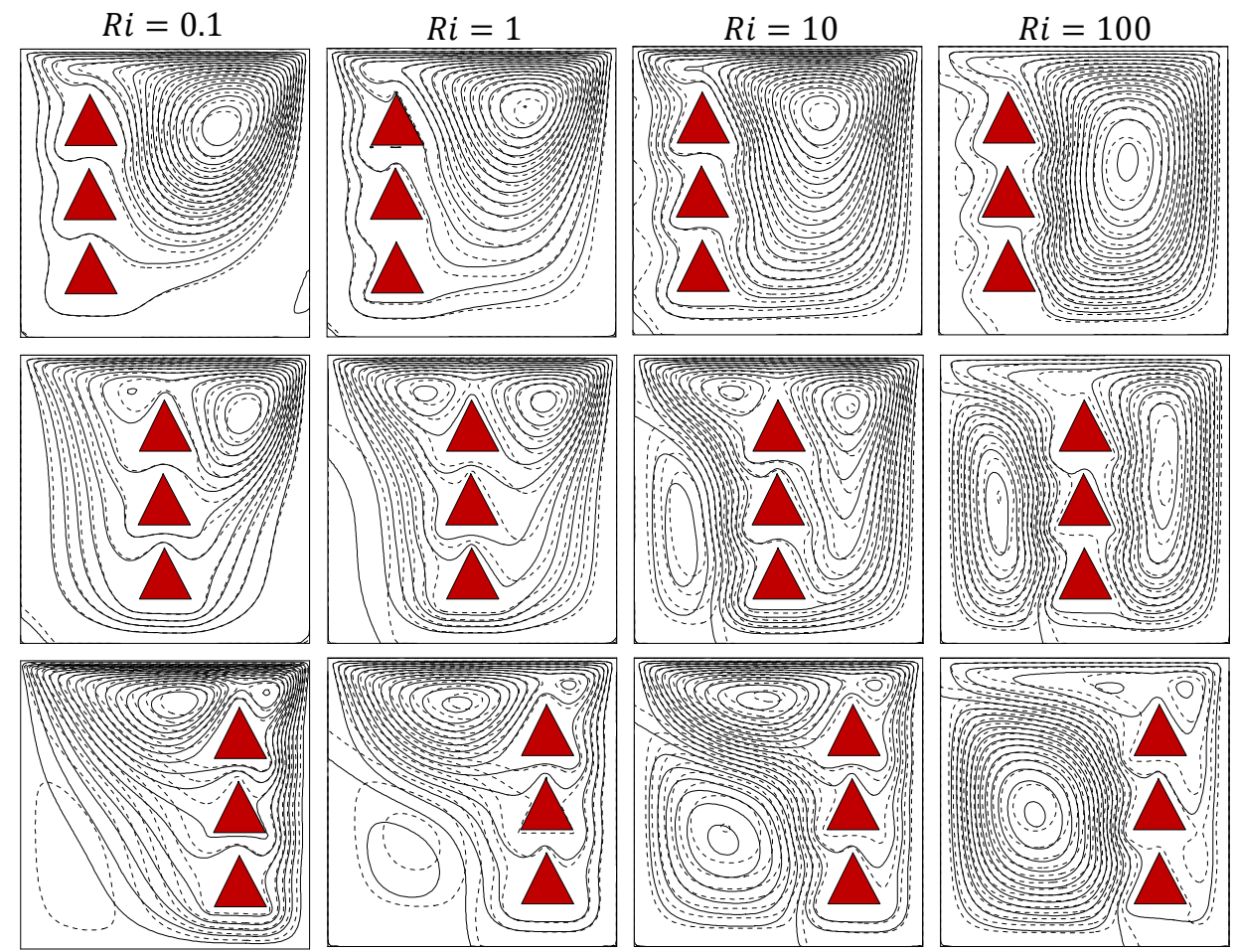

(a) streamlines
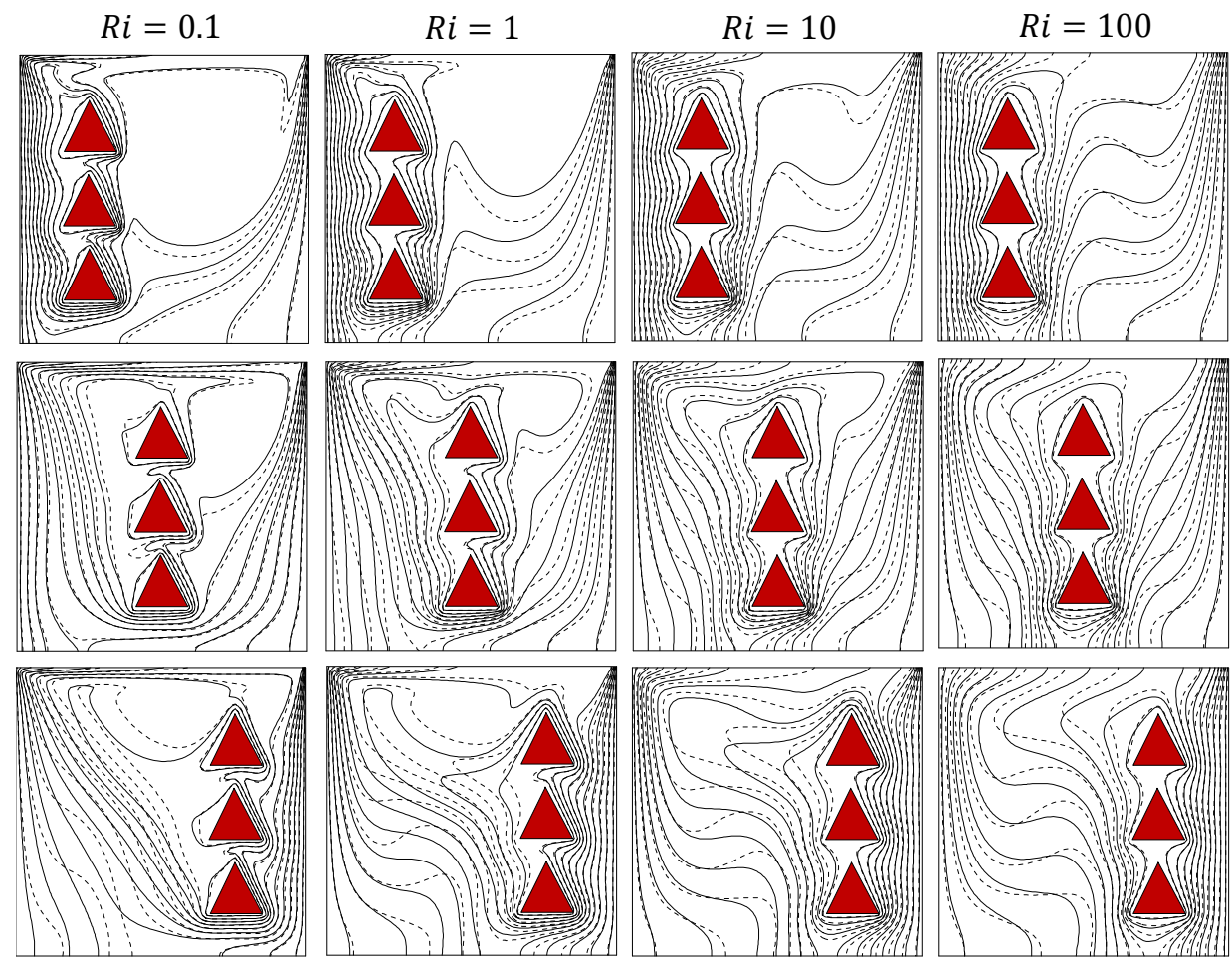

(b) isotherms

Fig. 5. (a) Streamlines and (b) isotherms inside the cavity corresponding to different locations of triangular blocks, i.e. $X=0.25,0.5$ and 0.75 filled with $\mathrm{Cu}$-water the pure fluid (dashed line) and $\mathrm{Cu}$-water nanofluid (solid line) with $\varphi=$ 5\% and at different Ris. Gr $=10^{4}$.

Fig. 6 shows values of $\Psi_{\max }$ and $\overline{\mathrm{Nu}}_{\text {tot }}$ in the same conditions of Fig. 5 at different blocks locations, Richardson number and volume fraction of nanoparticles. According to Fig. 6(b), it is noticed that by increasing volume fraction of nanoparticles and decreasing $R i$ the heat transfer rate increases. At all Richardson number, the heat transfer rate varies significantly among the different locations of the triangular blocks. This variation is due to the variation of the forced as well as natural convection effects among those different locations configurations. It is clear that the most preferable heat transfer is obtained when the triangular blocks are placed on the left side of the cavity. 

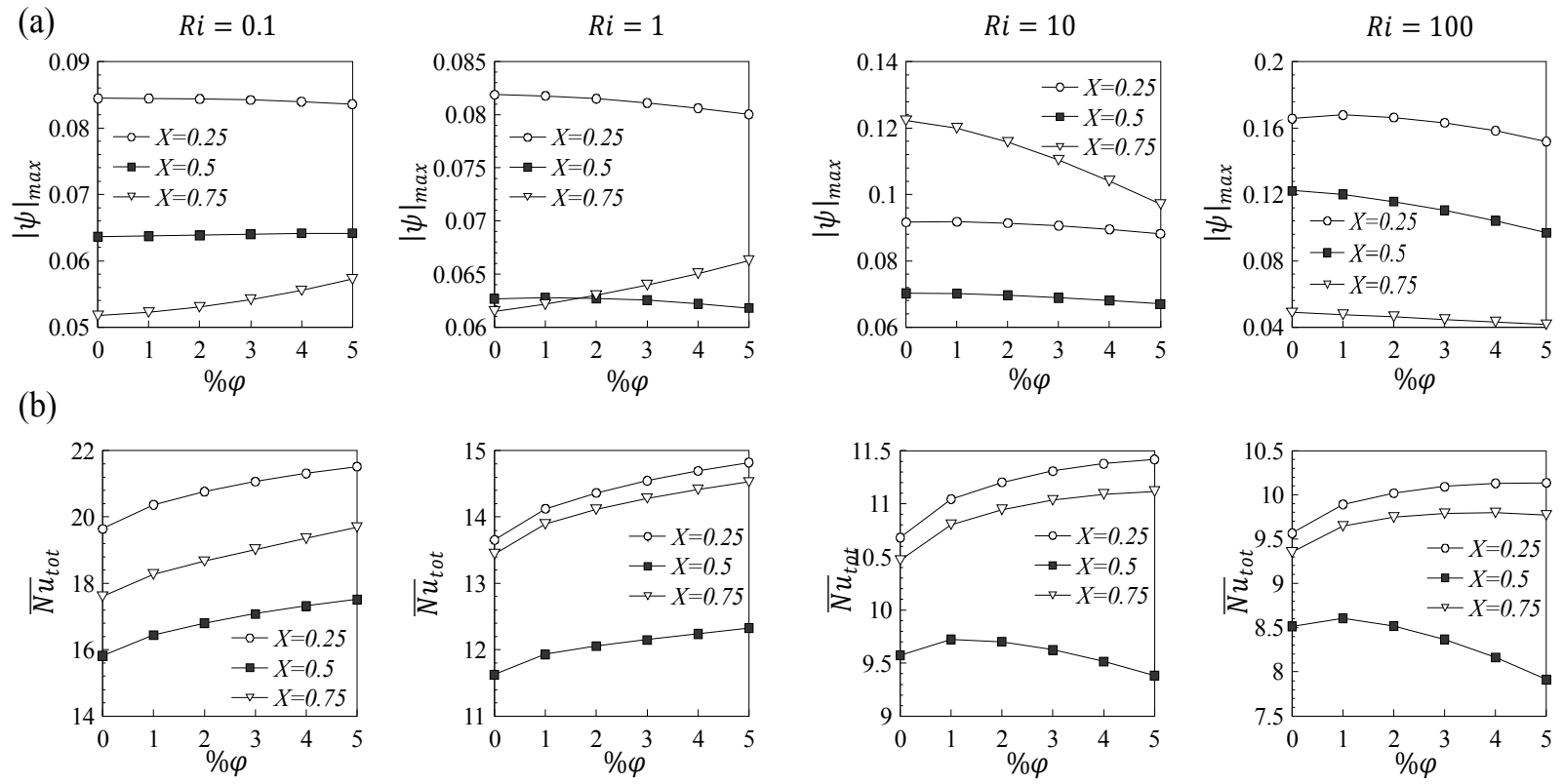

Fig. 6. Variation of (a) $\psi_{\max }$ and (b) $\overline{N u}_{t o t}$ corresponding to different locations of triangular blocks, i.e. $X=0.25,0.5$ and 0.75 for different Richardson numbers and volume fraction of the nanoparticles. $G r=10^{4}$.

\section{CONCLUSION}

Mixed convection heat transfer of nanofluid in a lid driven square cavity with three triangular heating blocks has been investigated. The calculations are carried out in two parts. First, the effect of vertical displacement of three triangular heating blocks $(0.25 \leq \mathrm{Y} \leq 0.75)$ is investigated. In the second part, the calculations are performed for the horizontal displacement of three triangular heating blocks $(0.25 \leq \mathrm{X} \leq$ $0.75)$ while the Richardson number $(R i)$ is ranging from 0.1 to 100 and the volume fraction of the nanoparticles is varying from 0 to 0.05 . According to the presented results, the following conclusions are drawn:

i. By decreasing the Richardson number and increasing the volume fraction of nanoparticles the rate of convective heat transfer is increased.

ii. When the three triangular heating blocks were arranged horizontally and positioned vertically from bottom to top $(0.25 \leq Y \leq 0.75)$. The maximum Nusselt number occurs when the triangular blocks are placed on the middle of the cavity $(Y=0.5)$, where there is more space between the triangular blocks and horizontal walls of the cavity.

iii. At high Richardson number $(R i \geq 10)$ the effects of natural convection are dominant so there is an optimum volume fraction of nanoparticles which maximize the heat transfer is about $1 \%$ in the most cases.

iv. When the three triangular heating blocks were arranged vertically and positioned horizontally from left to right $(0.25 \leq X \leq 0.75)$. The most preferable heat transfer is obtained when the triangular blocks are placed on the left side of the cavity.

\section{NOMENCLATURE}

$\begin{array}{ll}C_{p} & \text { specific heat, } \mathrm{Jkg}^{-1} \mathrm{~K}^{-1} \\ d_{p} & \text { diameter of the nanoparticle, } m \\ d_{f} & \text { diameter of the base fluid molecule, } m\end{array}$

$\begin{array}{ll}g & \text { gravitational acceleration, } m \mathrm{~s}^{-2} \\ \mathrm{Gr} & \text { Grashof number }\left(=g \beta \Delta T H^{3} / v^{2}\right) \\ H & \text { enclosure height, } \mathrm{m} \\ k & \text { thermal conductivity, } W \mathrm{~m}^{-1} \mathrm{~K}^{-1} \\ k_{b} & \text { Boltzmann's constant }=1.38066 \times 10^{-23} \\ N & \text { Number of triangular heated blocks } \\ \overline{N u}_{t o t} & \text { heat transfer of cavity's wall } \\ p & \text { dimensional pressure, } \mathrm{Nm}^{-2} \\ P & \text { dimensionless pressure } \\ P r & \text { Prandtl number }\left(=v_{f} / \alpha_{f}\right) \\ R a & \text { Rayleigh number }\left(=g \beta_{f}\left(T_{h}-T_{c}\right) H^{3} / \alpha_{f} v_{f}\right) \\ R e_{B} & \text { Brownian-motion Reynolds number } \\ R e & \text { Reynolds number }\left(=U_{0} H / v\right) \\ R i & \text { Richardson number }\left(=G r / R e^{2}\right) \\ T & \text { temperature, } K \\ t & \text { time, s } \\ \tau & \text { dimensionless time }\left(t / H / U_{r e f}\right) \\ u, v & \text { velocity components, } m s^{-1} \\ u_{B} & \text { Brownian velocity of the nanoparticle, } m s^{-1} \\ U, V & \text { dimensionless velocity components } \\ w & \text { dimensional width of the triangular heater } \\ W & \text { dimensionless width of the triangular heater } \\ x, y & \text { Cartesian coordinates, } m \\ X, Y & \text { dimensionless Cartesian coordinates }(x, y) / H\end{array}$

Greek symbols

$\begin{array}{ll}\alpha & \text { thermal diffusivity, } \mathrm{m}^{2} \mathrm{~s}^{-1} \\ \beta & \text { thermal expansion coefficient, } K^{-1} \\ \theta & \text { dimensionless temperature } \\ \mu & \text { dynamic viscosity, } \mathrm{kg} \mathrm{m}^{-1} \mathrm{~s}^{-1} \\ \nu & \text { kinematic viscosity, } \mathrm{m}^{2} \mathrm{~s}^{-1} \\ \rho & \text { density, } \mathrm{kgm}^{-3}\end{array}$


volume fraction of the nanoparticles

$\psi$

stream function $\left(=-\int_{Y_{o}}^{Y} U \partial Y+\psi\left(X, Y_{0}\right)\right)$

$\begin{array}{ll}\text { Subscripts } & \\ c & \text { cold } \\ f & \text { fluid } \\ h & \text { hot } \\ n f & \text { nanofluid } \\ s & \text { solid nanoparticles }\end{array}$

\section{ACKNOWLEDGMENTS}

The authors wish to express their very sincerely thanks to the reviewers for their valuable and lucid comments which have improved the paper appreciably.

\section{REFERENCES}

[1] M. Kalteh, K. Javaherdeh, T. Azarbarzin, Numerical solution of nanofluid mixed convection heat transfer in a lid-driven square cavity with a triangular heat source, Powder Technol. 253 (2014) 780-788..

[2] A.W. Islam, M.a.R. Sharif, E.S. Carlson, Mixed convection in a lid driven square cavity with an isothermally heated square blockage inside, Int. J. Heat Mass Transf. 55 (2012) 5244-5255.

[3] K. Khanafer, S.M. Aithal, Laminar mixed convection flow and heat transfer characteristics in a lid driven cavity with a circular cylinder, Int. J. Heat Mass Transf. 66 (2013) 200-209.

[4] H.F Oztop, I. Dagtekin, A. Bahloul, Comparison of position of a heated thin plate located in a cavity for natural convection, Int. Commun. Heat Mass Transfer 31 (2004) 121-132.

[5] Sheikholeslami M, Gorji-Bandpy M, Vajravelu K. Lattice Boltzmann simulation of magnetohydrodynamic natural convection heat transfer of $\mathrm{Al}_{2} \mathrm{O}_{3}$-water nanofluid in a horizontal cylindrical enclosure with an inner triangular cylinder. Int $\mathrm{J}$ Heat Mass Transfer 2015;80:16-25.

[6] M. El Abdallaoui, M. Hasnaoui and A. Amahmid, Numerical simulation of natural convection between a decentered triangular heating cylinder and a square outer cylinder filled with a pure fluid or a nanofluid using the lattice Boltzmann method, Powder Technol. 277 (2015) 193-205

[7] M. El Abdallaoui, M. Hasnaoui and A. Amahmid, Lattice-Boltzmann modeling of natural convection between a square outer cylinder and an inner isosceles triangular heating body, Num. Heat Transfer, Part A, vol. 66, pp. 1076-1096, 2014.

[8] H.F. Oztop, Z. Zhao, B. Yu, Fluid flow due to combined convection in lid-driven enclosure having circular body, Int. J. Heat Fluid Flow 30 (2009) 886-901.

[9] I. Pishkar, B. Ghasemi, Cooling enhancement of two fins in a horizontal channel by nanofluid mixed convection, Int. J. Therm. Sci. 59 (2012) 141-151.
[10] M. Corcione, Empirical correlating equations for predicting the effective thermal conductivity and dynamic viscosity of nanofluids, Energy Convers. Manag. 52 (2011) 789-793

[11] Z. Haddad, H.F. Oztop, E. Abu-Nada, A. Mataoui, A review on natural convective heat transfer of nanofluids, Renewable Sustainable Energy Rev. 16 (2012) 53635378.

[12] M. Corcione, Heat transfer features of buoyancy-driven nanofluids inside rectangular enclosures differentially heated at the sidewalls, Int. J. Therm. Sci. 49 (2010) $1536-1546$

[13] R. Iwatsu, J.M. Hyun, K. Kuwahara, Mixed convection in a driven cavity with a stable vertical temperature gradient, Int. J. Heat Mass Transfer 36 (1993) 16011608.

[14] H. Moumni, H. Welhezi, R. Djebali, E. Sediki, Accurate finite volume investigation of nanofluid mixed convection in two-sided lid driven cavity including discrete heat sources, Appl. Math. Model. (2014).

[15] C.L. Chen, S.C. Chang, C.K. Chen, C.K. Chang, Lattice boltzmann simulation for mixed convection of nanofluids in a square enclosure, Appl. Math. Model. 39 (2015) 2436-2451

[16] Z. Boulahia, A. Wakif, and R. Sehaqui, "Natural Convection Heat Transfer of the nanofluids in a Square Enclosure with an Inside Cold Obstacle," International Journal of Innovation and Scientific Research, vol. 21, no. 2, pp. 367-375, April 2016

[17] M. Muthtamilselvan, D.H. Doh, Mixed convection of heat generating nanofluid in a lid-driven cavity with uniform and non-uniform heating of bottom wall, Appl. Math. Model. 38 (2014) 3164-3174.

[18] Z. Boulahia and R. Sehaqui, "Numerical Simulation of Natural Convection of Nanofluid in a Square Cavity Including a Square Heater", International Journal of Science and Research (IJSR), ijsr.net, Volume 4 Issue 12, December 2015, 1718 - 1722

[19] D.B. Spalding, A novel finite difference formulation for differential expressions involving both first and second derivatives, Int. J. Numer. Methods Eng. 4 (1972) 551559 .

[20] Z. Boulahia, A. Wakif, and R. Sehaqui, "Numerical Study of Mixed Convection of the Nanofluids in TwoSided Lid-Driven Square Cavity with a Pair of Triangular Heating Cylinders," Journal of Engineering, vol. 2016, Article ID 8962091, 8 pages, 2016.

[21] F.P. Incropera, D.P. DeWitt, Introduction to Heat Transfer, Wiley, New York, 2002.

[22] S.V. Patankar, Numerical Heat Transfer and Fluid Flow, McGraw-Hill, Washington, 1980. 